KANSAS JOURNAL of MEDICINE

\section{Guideline Adherence in Dyspepsia Investigation and Treatment}

Katelyn Dugan, MS-3ㄹ, Elizabeth Ablah, Ph.D., MPH',

Hayrettin Okut, Ph.D., ${ }^{1,2}$, Sachin Srinivasan, M.D. ${ }^{3}$,

William Salyers, Jr., M.D., MPH ${ }^{3}$

University of Kansas School of Medicine-Wichita, Wichita, KS

'Department of Population Health

${ }^{2}$ Office of Research

${ }^{3}$ Department of Internal Medicine

Received July 1, 2020; Accepted for publication Aug. 20, 2020; Published online Dec. 11, 2020 https:/ doi.org 10.17161 lijm.voll3.13838

\section{ABSTRACT}

Introduction. The impact of dyspepsia guidelines on clinical practice may be poor. Provider adherence with dyspepsia guidelines was examined to determine their impact on clinical practice.

Methods. Provider adherence with the 2005 American College of Gastroenterology Guidelines for the Management of Dyspepsia and the 2017 American College of Gastroenterology and Canadian Association of Gastroenterology joint Dyspepsia Management Guidelines was assessed on a national level using data from the National Ambulatory Medical Care Survey (NAMCS). Patient visit data, including reason for visit of dyspepsia, diagnosis of dyspepsia, or diagnosis of H. pylori infection from NAMCS years 2012 through 2015, were used. Provider adherence with dyspepsia management guidelines was determined based upon provision of at least one recommended test or treatment for dyspepsia.

Results. Providers appeared to adhere to the 2005 ACG guidelines for $49.7 \%$ of patient visits. Providers appeared to adhere to the 2017 ACG/CAG guidelines for $51.0 \%$ of patient visits.

Conclusion. Provider adherence with the 2005 ACG and the 2017 ACG/CAG Dyspepsia Management Guidelines was determined to be low in this study, highlighting the need to increase evidence-based medical treatment and efficient resource use for dyspepsia.

\section{Kans J Med 2020;13:306-310 \\ INTRODUCTION}

Dyspepsia has been described as epigastric pain persisting for one month or more, which may be associated with a range of other clinical gastrointestinal symptoms, including heartburn or epigastric fullness. ${ }^{1}$ The prevalence of uninvestigated dyspepsia in North America is approximately $22 \%$, with a suggested incidence of $1.3 \%$ annually and peak incidence in the seventh decade of life.,3

Dyspepsia patients can be categorized as (1) uninvestigated if they have not undergone investigation in an attempt to discover underlying disease, (2) investigated, which exposed a cause for their symptoms, or (3) investigated, but no cause was discovered, termed functional dyspepsia, a diagnosis of exclusion. ${ }^{4}$ Investigation by endoscopy revealed clinically significant findings in $10.2 \%$ of patients, with one study suggesting nonerosive gastritis and another suggesting reflux esophagitis most commonly, were identified., ${ }^{5,6}$ Other underlying causes of dyspepsia included infection of Helicobacter pylori (H. pylori), gastric or duodenal ulcers, and gastrointestinal malignancy, although functional dyspepsia is the most common cause.,7

Different tests and procedures may be done to investigate dyspepsia symptoms, some of which are not recommended routinely and may increase healthcare costs for dyspepsia management. Among patients diagnosed with functional dyspepsia, the cost of testing received was estimated to be $\$ 582$ per patient. ${ }^{8}$ These patients underwent blood work (75\%), abdominal ultrasound (59\%), abdominal x-ray (47\%), or abdominal and/or pelvis computed tomography (CT; 40\%). ${ }^{8}$ None of these tests are recommended routinely for evaluation of dyspepsia by either the 2005 American College of Gastroenterology clinical guidelines or the 2017 American College of Gastroenterology and Canadian Association of Gastroenterology joint guidelines. ${ }^{1,9}$

The impact of dyspepsia guidelines on clinical practice may be poor. A vignette survey suggested $57 \%$ of primary care providers and $74 \%$ of gastroenterologists adhered to the 2005 ACG guidelines and other consensus recommendations for dyspepsia investigation and management. ${ }^{10}$

Endoscopy may be overutilized to investigate dyspepsia. One study suggested $37.8 \%$ of esophagogastroduodenoscopies (EGD) done for gastrointestinal indications, including dyspepsia, were not indicated by guidelines. ${ }^{11}$ A study of dyspepsia patients younger than 55 years without alarm symptoms suggested 58\% (rather than 100\%) had received both $H$. pylori testing and proton pump inhibitors (PPI) therapy, as the guidelines recommended, before receiving an EGD. ${ }^{12}$ However, endoscopy may be underutilized in older age groups, as one study reported $81 \%$ of dyspepsia patients 50 years or older had not received endoscopy, despite guideline recommendations. ${ }^{13}$ Another study reported similar results; only $27 \%$ of dyspepsia patients older than 55 years had received an EGD, rather than $100 \%$ if the 2005 guidelines had been followed. ${ }^{3}$

The current study examined dyspepsia investigation and treatment practices for 2012 - 2015. Provider practices were determined to be adherent or nonadherent with the 2005 ACG Guidelines for the Management of Dyspepsia. Adherence with the 2017 ACG and CAG guidelines also was assessed to determine if clinical practices in the years preceding the release of these guidelines had shifted toward utilizing therapies included in the 2017 guidelines, as new knowledge on effective management was identified, but before updated guidelines were released.

\section{METHODS}

Participants. Data were analyzed from the National Ambulatory Medical Care Survey (NAMCS) for 2012 - 2015..$^{14}$ Data collected about community health centers were not included. Patient data meeting the following criteria were included: reason(s) for visit including dyspepsia, diagnosis of dyspepsia, or diagnosis of Helicobacter pylori infection. Data were excluded if patients were younger than 18 years, or if the patient had a diagnosis of gastroesophageal reflux disease (GERD).

Instrument. Non-federally employed, practicing physicians (excluding pathologists, radiologists, and anesthesiologists) working 
in ambulatory care settings in the U.S. were eligible to participate in NAMCS. Physician interviews were used to collect physician and healthcare practice data (e.g., specialty, location). Physicians were surveyed for a sample of patient visits during a random oneweek period annually. Data recorded about patient visits included demographic information, patient comorbidities, reason(s) for visit, diagnoses related to the visit, services ordered or provided, medications (including prescription and over-the-counter drugs) ordered or provided, and if these medications were new or continued. Drug information, including drug therapeutic classes, for medications ordered or provided during the patient visit, was coded by NAMCS using Lexicon Plus ${ }^{\circledast}$, a Cerner Multum ${ }^{\mathrm{TM}}$, Inc. database. ${ }^{15}$

The variables used from the NAMCS database files for the current study included patient age, body mass index (BMI), sex, unimputed race, unimputed ethnicity, tobacco use, depression comorbidity, reason for visit, diagnoses related to visit, services ordered or provided, and therapeutic class codes for medications ordered or provided. Up to five reasons for visit and five diagnoses could be listed per patient record. For services not specifically listed as a checkbox item on the patient record interview form, up to nine write-in procedure codes could be documented. Up to 10 medications could be listed for years 2012 and 2013, and up to 30 medications could be listed for years 2014 and 2015. For each medication, up to four categories, with three levels per category, could be used to list the drug's therapeutic class codes from the Lexicon Plus ${ }^{\boxplus}$ database. $^{15}$

Data about the physician, including specialty and region of practice within the U.S., were used. Physician specialty was stratified into 14 groups, including general/family practice, internal medicine, and general surgery. Physician specialty was stratified separately into three groups: primary care, surgical care, or medical care specialties. Regions of practice included the northeast, midwest, south, and west regions of the U.S.

Procedures. This study was considered "not human subjects" by the University of Kansas School of Medicine-Wichita's Human Subjects Committee. To assess the frequency of medications ordered or prescribed, drug therapeutic class codes were used. Drug therapeutic classes of interest included antacids, PPIs, $\mathrm{H} 2$ antagonists, $H$. pylori eradication agents, gastrointestinal stimulants, and tricyclic antidepressants (TCA).

The frequency of a diagnosis of $H$. pylori infection (ICD-9-CM code 041.86) along with one or more write-in procedure codes encompassing noninvasive $H$. pylori testing recommended by the 2007 ACG H. pylori management guidelines was used to determine the frequency H. pylori tests were ordered or $\operatorname{provided}^{16}$ (Table 1).

Table 1. Patient characteristics.

\begin{tabular}{|l|l|}
\hline 89.39 & $\begin{array}{l}\text { Other nonoperative measurements and examinations (includ- } \\
\text { ing 14 C-Urea breath test). }\end{array}$ \\
\hline 90.59 & Other microscopic examination of blood. \\
\hline 90.99 & $\begin{array}{l}\text { Other microscopic examination of specimen from lower gas- } \\
\text { trointestinal tract and of stool. }\end{array}$ \\
\hline 91.39 & $\begin{array}{l}\text { Other microscopic examination of specimen from bladder, } \\
\text { urethra, prostate, seminal vesicle, perivesical tissue, and of } \\
\text { urine and semen. }\end{array}$ \\
\hline
\end{tabular}

KANSAS JOURNAL of MEDICINE DYSPEPSIA GUIDELINEADHERENCE

continued.

To assess the frequency of EGD or other upper gastrointestinal endoscopy ordered or provided during a patient visit, data from the checkbox item "upper gastrointestinal endoscopy/EGD” for years 2014 and 2015 were combined with write-in upper endoscopy ICD-9-CM procedure codes for years 2012 through 2015 (Table 2). The combined frequency of the EGD checkbox item and the upper endoscopy procedure codes was used to assess the number of upper endoscopies ordered or performed.

Table 2. Write-in upper endoscopy ICD-9-CM procedure codes.

\begin{tabular}{|l|l|}
\hline 42.23 & Other esophagoscopy (excludes that with biopsy). \\
\hline 42.24 & Closed [endoscopic] biopsy of esophagus. \\
\hline 44.13 & Other gastroscopy (excludes that with biopsy). \\
\hline 44.14 & Closed [endoscopic] biopsy of stomach. \\
\hline 45.13 & Other endoscopy of small intestine (includes EGD). \\
\hline 45.16 & EGD with closed biopsy. \\
\hline
\end{tabular}

To assess provider adherence with the 2005 ACG guidelines, the provision of at least one component of the 2005 guidelines constituted adherence. Specifically, the provision of any upper endoscopy/EGD, acid suppression medication (PPIs), H. pylori eradication agents, or noninvasive $H$. pylori testing was assessed per patient to determine provider guideline adherence (Figure 1). To assess provider adherence with the $2017 \mathrm{ACG} / \mathrm{CAG}$ guidelines, the provision of at least one component of the 2017 guidelines constituted adherence. All components of the 2005 guidelines were included in the 2017 assessment, with the addition of tricyclic antidepressant medications, GI stimulant/prokinetic medications, or psychotherapy (Figure 1). H2 antagonists were included as acceptable acid suppression therapy for adherence with the 2005 and 2017 guidelines in two additional assessments of provider adherence.

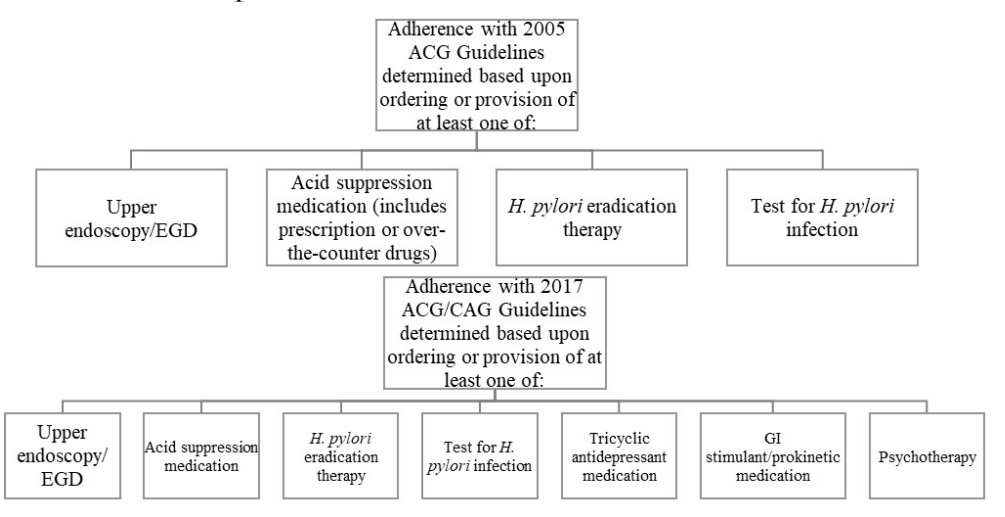

Figure 1. Criteria for guideline adherence.

Analysis. Data were analyzed using SAS version 9.4 (2019, SAS Int. Inc., Carry, NC). Frequencies, proportion, means, and standard deviations were calculated. Pearson chi-square and likelihood chi-square were used to test the associations between categorical variables. Cochran-Mantel-Haenszel statistics were conducted to determine the relationships between two stratified categorical variables after controlling for at least one variable. These stratified analyses 
KANSAS JOURNAL of MEDICINE DYSPEPSIA GUIDELINE ADHERENCE

continued.

provided a way to adjust for the possible confounding effects without being forced to estimate parameters for them. All statistical tests at $p$ $\leq 0.05$ were considered significant.

\section{RESULTS}

Of the 260,118 patient encounters from 2012 through 2015, 680 records met inclusion/exclusion criteria. Of the 680 records, heartburn and indigestion (dyspepsia; ICD-9-CM code 1535.0) was documented as the reason for $58.5 \%$ of patient visits $(n=398)$. The diagnosis of dyspepsia or other specified disorders (ICD-9-CM code $536.8)$ was listed for $38.2 \%$ of patients $(\mathrm{n}=260)$, and the diagnosis of H. pylori infection (ICD-9-CM code 041.86) was listed for $13.1 \%$ of patients $(\mathrm{n}=89)$.

Physician participants worked throughout the U.S., with $34.4 \%$ practicing primarily in southern states $(\mathrm{n}=234), 23.1 \%$ in western states $(n=157), 23.1 \%$ in the midwest $(n=157)$, and $19.4 \%$ in the northeast $(\mathrm{n}=132)$. Broadly grouped, $49.0 \%$ of physicians $(\mathrm{n}=333)$ were in medical care specialties, $42.2 \%$ of physicians $(n=287)$ were in primary care specialties, and $8.8 \%$ of physicians $(n=60)$ were in surgical care specialties. Of the 14 specialty groupings, many physicians $(41.0 \%, \mathrm{n}=194)$ were categorized into the "other" specialties group, which included gastroenterology. Physicians in the "general or family practice" group made up $20.9 \%$ of participants $(\mathrm{n}=99)$, and those in internal medicine made up another $20.9 \%$ of participants $(n=99)$.

As reported by their physicians, most patients $(82.3 \%, \mathrm{n}=415)$ were White, and $9.9 \%$ of patients $(n=50)$ were Black or African American. Almost $20 \%$ of patients $(18.2 \%, \mathrm{n}=88)$ were reported to be Hispanic or Latino (Table 3).

Table 3. Patient demographics.

\begin{tabular}{|l|c|c|}
\hline & Frequency & Percent \\
\hline Sex & & \\
\hline Female & 411 & $60.4 \%$ \\
\hline Male & 269 & $39.6 \%$ \\
\hline Ethnicity & & \\
\hline Not Hispanic/Latino & 396 & $81.8 \%$ \\
\hline Hispanic/Latino & 88 & $18.2 \%$ \\
\hline Missing & 196 & \\
\hline Race & & \\
\hline White/Caucasian & 415 & $82.3 \%$ \\
\hline Black/African American & 50 & $9.9 \%$ \\
\hline Asian American & 33 & $6.5 \%$ \\
\hline More than I Race Reported & 3 & $0.6 \%$ \\
\hline Native Hawaiian/Other Pacific Islander & 2 & $0.4 \%$ \\
\hline American Indian/Alaskan Native & 1 & $0.2 \%$ \\
\hline Missing & 176 & \\
\hline
\end{tabular}

Patient mean age was 55 years, and $60.4 \%$ of patients $(n=411)$ were female. Mean patient BMI $(n=521)$ was 29 . Physicians reported that $16.5 \%$ of patients $(\mathrm{n}=90)$ were current tobacco users and $10.6 \%$ of patients $(\mathrm{n}=72)$ had current depression.

Almost all patients $(91.8 \%, \mathrm{n}=624)$ had at least one service ordered or provided, including exams, screenings, laboratory tests, imaging, procedures, treatments, health education, or counseling. More than one quarter of physicians $(26.3 \%, \mathrm{n}=179)$ reported having one or more exams ordered or provided. Almost one in five patients (18.5\%, $\mathrm{n}=126$ ) had some type of imaging ordered or provided, including an $\mathrm{x}$-ray for $7.2 \%$ of patients $(\mathrm{n}=49)$, CT scan for $4.9 \%$ of patients $(\mathrm{n}=$ $33)$, or magnetic resonance imaging for $1.3 \%$ of patients $(n=9)$.

One or more write-in procedures were reported for $43.4 \%$ of patients $(n=295)$, for a total of 486 write-in procedures. Procedures included "other microscopic examination of blood" $(17.5 \%, \mathrm{n}=85)$, upper endoscopy $(13.8 \%, \mathrm{n}=67)$, "other consultation" $(10.1 \%, \mathrm{n}=$ $49)$, and general physical examination $(3.5 \%, \mathrm{n}=17)$.

Of prescription and non-prescription medications reported, antacids were ordered or provided for $3.8 \%$ of patients $(n=26)$. Of the acid suppression medications, PPIs $(41.3 \%, \mathrm{n}=281)$ were ordered or provided more often than $\mathrm{H} 2$ antagonists $(7.9 \%, \mathrm{n}=54)$. TCAs were ordered or provided for $1.8 \%$ of patients $(n=12)$, and GI stimulants/ prokinetics were ordered or provided for $1.6 \%$ of patients $(n=11)$. Psychotherapy was ordered or provided for $0.6 \%$ of patients $(n=4)$.

Noninvasive $H$. pylori testing was ordered or provided for $2.9 \%$ of patients $(\mathrm{n}=20)$. H. pylori eradication therapy was given for $1.6 \%$ of patients $(\mathrm{n}=11)$. Any EGD/upper endoscopy was ordered or provided for $12.8 \%$ of patients $(n=87$; Table 4$)$.

Table 4. Management provided.

\begin{tabular}{|l|c|c|}
\hline & Frequency & Percent \\
\hline \multicolumn{2}{|c|}{ Medications (Prescription and Non-Prescription) } \\
\hline Proton Pump Inhibitors (PPIs) & 281 & $41.3 \%$ \\
\hline H2 Antagonists & 54 & $7.9 \%$ \\
\hline Antacids & 26 & $3.8 \%$ \\
\hline Tricyclic Antidepressants (TCA) & 12 & $1.8 \%$ \\
\hline GI Stimulants/Prokinetics & 11 & $1.6 \%$ \\
\hline H. pylori Testing or Treatment & 20 & $2.9 \%$ \\
\hline Noninvasive H.pylori Test & 11 & $1.6 \%$ \\
\hline H. pylori Eradication Therapy & 87 & $12.8 \%$ \\
\hline EGD/Upper Endoscopy & \multicolumn{2}{|l}{} \\
\hline EGD/Upper Endoscopy &
\end{tabular}

When adherence was defined as provision of any component of the 2005 ACG dyspepsia guidelines, providers were considered adherent for $49.7 \%(n=338)$ of patient visits. When adherence was defined as provision of any component of the $2017 \mathrm{ACG} / \mathrm{CAG}$ dyspepsia guidelines, providers were considered adherent for $51.0 \%(\mathrm{n}=347)$ of patient visits (Table 5). Addition of $\mathrm{H} 2$ antagonists for acceptable acid suppression raised adherence to $54.1 \%$ of patient visits $(\mathrm{n}=368)$ for the 2005 ACG guidelines and $55.4 \%$ of patient visits $(n=377)$ for the 2017 ACG/CAG guidelines. 
Table 5. Guideline adherence.

\begin{tabular}{|c|c|c|}
\hline & Frequency & Percent \\
\hline \multicolumn{3}{|l|}{2005 ACG Guideline Criteria } \\
\hline Total Adherence from 2012 through 2015 & 338 & $49.7 \%$ \\
\hline Adherence in 2012 & 95 & $45.9 \%$ \\
\hline Adherence in 2013 & 152 & $53.1 \%$ \\
\hline Adherence in 2014 & 67 & $52.8 \%$ \\
\hline Adherence in 2015 & 24 & $40.0 \%$ \\
\hline \multicolumn{3}{|l|}{2017 ACG/CAG Guideline Criteria } \\
\hline Total Adherence from 2012 through 2015 & 347 & $51.0 \%$ \\
\hline Adherence in 2012 & 95 & $45.9 \%$ \\
\hline Adherence in 2013 & 154 & $53.8 \%$ \\
\hline Adherence in 2014 & 72 & $56.7 \%$ \\
\hline Adherence in 2015 & 26 & $43.3 \%$ \\
\hline
\end{tabular}

\section{DISCUSSION}

The prevalence of dyspepsia in this study was $0.26 \%(n=680)$. A prior study suggested the prevalence of gastritis/dyspepsia to be $1.73 \%$ among adults. ${ }^{17}$ The lower prevalence of dyspepsia within this study may be due to the strict inclusion criteria. It is likely that some patients presenting with dyspepsia were not reported as such by physicians during the patient record interview, and instead another reason for visit or diagnosis was used, such as upper abdominal pain (ICD-9-CM code 1545.3 upper abdominal pain, cramps, spasms) or diagnosis of gastric ulcer (ICD-9-CM code 531 Gastric Ulcer). The code for upper abdominal pain was not used for inclusion criteria in this study, as it encompasses more than just epigastric pain and likely would have overestimated the prevalence of dyspeptic patients within the study sample. Likewise, more specific diagnoses, such as gastric ulcers or gastrointestinal malignancy, were not used for inclusion criteria in this study, as they are considered underlying causes of dyspepsia identified on EGD which may require separate therapy. ${ }^{18,19}$

Diagnosis of $H$. pylori infection was used for inclusion criteria in this study, as the 2007 ACG Guideline on the Management of Helicobacter pylori Infection recommended diagnosis and treatment for select patients with uninvestigated dyspepsia. ${ }^{16}$ Although diagnosis of $H$. pylori is indicated for reasons other than dyspepsia (e.g., peptic ulcer disease, gastric mucosa associated lymphoid tissue or MALT lymphoma), the diagnosis of $H$. pylori was used to identify a small proportion (13.1\%) of the study sample and is unlikely to have a large impact on the results. ${ }^{16}$ The diagnosis of GERD was used for exclusion, as GERD is considered a different diagnosis for patients presenting mainly with heartburn or acid regurgitation, rather than dominant epigastric discomfort or pain as in dyspepsia. ${ }^{9}$

The current study suggested dyspepsia to be more common among women. Uninvestigated dyspepsia may be more common among women, with a suggested global prevalence of $25.3 \%$ in women compared to $21.9 \%$ among men. ${ }^{2}$ Patient mean age in this study was 55 years, which is slightly older than the mean ages of 47 years and 50 years of two other studies of patients with dyspepsia. ${ }^{8,20}$

In the current study, $18.5 \%$ of patients $(n=126)$ were ordered or provided an imaging service. A study of patients with persistent dyspepsia suggested that $87.1 \%$ of patients had received abdominal imaging, and
KANSAS JOURNAL of MEDICINE

DYSPEPSIA GUIDELINEADHERENCE

continued.

the most frequent modalities were CT and ultrasound. ${ }^{21}$ The lower frequency of imaging use in the current study could indicate a decreasing trend as study data were obtained from more recent years, although history of prior or later imaging services provided to patients in this study was not obtainable.

The current study suggested that PPIs were prescribed more often than $\mathrm{H} 2$ antagonists. Physician concern about adverse effects of PPI therapy have been suggested, with $52 \%$ of internal medicine physicians self-reporting they sometimes or often changed a patient's prescription from PPI therapy to $\mathrm{H} 2$ antagonists. ${ }^{22}$ A prior study suggested $\mathrm{H} 2$ antagonists were prescribed more frequently than PPIs in patients with uninvestigated dyspepsia ( $88.1 \%$ versus $47.4 \%$, respectively). ${ }^{13}$ However, the current study's findings were consistent with guideline recommendations, and this increased usage of PPIs over $\mathrm{H} 2$ antagonists for acid suppression therapy may indicate guideline adherence in this area. ${ }^{1,9}$

Half of patients with dyspepsia in this study received at least one treatment, diagnostic test, or exam recommended by either the 2005 ACG or 2017 ACG/CAG guidelines. This percentage of patients receiving care specified within dyspepsia management guidelines was suboptimal and indicated low physician adherence to dyspepsia management guidelines. Furthermore, there was minimal difference between the percent of visits adherent to the 2005 compared to the 2017 guidelines, indicating the additional therapies considered adherent by the 2017 guidelines were not utilized frequently in the years preceding the release of the 2017 guidelines. This limited difference in adherence also clarified that most visits considered non-adherent with the 2005 guidelines were not provided the more novel therapies considered to be adherent by the 2017 guidelines.

Physicians may be non-adherent due to lack of knowledge of guidelines. A review of guideline adherence barriers suggested physicians were more likely to be unfamiliar with guideline content than unaware of their existence. ${ }^{23}$ Therefore, dissemination of the 2017 ACG/CAG guidelines with its management algorithm to physicians working in ambulatory care settings may improve knowledge and adherence to dyspepsia management guidelines. However, this strategy alone may not be sufficient to raise adherence levels, as passive dissemination of guidelines has been suggested to be ineffective for guideline implementation. ${ }^{24}$ Further interventions, including workshops, handouts, and reminders, may be beneficial as they have been suggested to lower drug costs of dyspepsia without increasing endoscopy use. ${ }^{25}$

Increased monitoring of guideline adherence in clinical practices may be beneficial. Notifying providers when their endoscopy referral for dyspepsia management was not adherent with guidelines was suggested to increase adherence rates from $55 \%$ to $75 \% .{ }^{26}$ Less targeted approaches to increase physician acceptance and adherence to all clinical guidelines may be useful, as one study suggested clinical practice guidelines only have a large or very large effect on how less than $25 \%$ of physicians practice medicine. ${ }^{27}$ Both targeted and general approaches 
KANSAS JOURNAL of MEDICINE

DYSPEPSIA GUIDELINEADHERENCE

continued.

to increase guideline adherence may be necessary to ensure evidence-

based medicine is practiced.

There were several limitations of this study. NAMCS is a voluntary survey of physicians. As a result, data may be influenced by response and/or recall bias. There was also variability in the number of records meeting inclusion/exclusion criteria per year. The highest number of patient records meeting criteria for this study was 286 records in 2013 and the lowest number was 60 in 2015. The lack of consistency in the total number of patient records available per year from the NAMCS database may have led to increased variability in the data. However, the percentage of records determined to be adherent to guidelines in this study remained low in each year, ranging from $40.0 \%$ to $53.1 \%$ for the 2005 guidelines and from $43.3 \%$ to $56.7 \%$ for the 2017 guidelines.

The lack of available CPT codes, the broadness of the ICD-9$\mathrm{CM}$ codes, and the variability in the checkbox items included in the survey from year to year made capturing accurate and consistent data difficult. For example, upper gastrointestinal endoscopy/EGD was included as a checkbox item for examinations/screenings ordered or provided for survey years 2014 and 2015, but not for years 2012 or 2013. Furthermore, appropriateness of the use of treatments or tests for each patient based upon specific indications (such as presence of alarm features, risk of malignancy, or previous treatment or test results) could not be assessed in this cross-sectional study. Additionally, some patients may have not desired or had counter-indications to receiving the guideline recommended test or treatment, making non-adherence acceptable in these specific situations. Therefore, adherence may have been over or underestimated, as the specific order and situational appropriateness of guideline provision could not be assessed.

Despite the challenges of using NAMCS data, the survey captured national, systematic data on ambulatory care practices within the U.S. It is a valuable source of information about clinical practices of U.S. physicians.

\section{CONCLUSIONS}

Provider adherence with both the 2005 ACG and the 2017 ACG/ CAG dyspepsia management guidelines was determined to be low in this study, with $50 \%$ of patients receiving a recommended test or treatment. Physician adherence must increase to evidence-based management of patients with dyspepsia and utilize healthcare resources appropriately and efficiently.

\section{REFERENCES}

${ }^{1}$ Moayyedi PM, Lacy BE, Andrews CN, Enns RA, Howden CW, Vakil N. ACG and CAG Clinical Guideline: Management of Dyspepsia. Am J Gastroenterol 2017; 112(9):1484. PMID: 28762378.

2 Ford AC, Marwaha A, Sood R, Moayyedi P. Global prevalence of, and risk factors for, uninvestigated dyspepsia: A meta-analysis. Gut 2015; 64(7):10491057. PMID: 25147201.

${ }^{3}$ Mapel D, Roberts M, Overhiser A, Mason A. The epidemiology, diagnosis, and cost of dyspepsia and Helicobacter pylori gastritis: A case-control analysis in the Southwestern United States. Helicobacter 2013; 18(1):54-65. PMID: 23067108.
${ }^{4}$ Black CJ, Houghton LA, Ford AC. Insights into the evaluation and management of dyspepsia: Recent developments and new guidelines. Therap Adv Gastroenterol 2018; 11:1756284818805597. PMID: 30397412.

5 Abdeljawad K, Wehbeh A, Qayed E. Low prevalence of clinically significant endoscopic findings in outpatients with dyspepsia. Gastroenterol Res Pract 2017; 2017:3543681. PMID: 28210269.

6 Thomson AB, Barkun AN, Armstrong D, et al. The prevalence of clinically significant endoscopic findings in primary care patients with uninvestigated dyspepsia: The Canadian Adult Dyspepsia Empiric Treatment - Prompt Endoscopy (CADET-PE) Study. Aliment Pharmacol Ther 2003; 17(12):14811491. PMID: 12823150

7 Talley NJ, Ford AC. Functional dyspepsia. N Engl J Med 2015; 373(19):1853-1863. PMID: 26535514.

${ }^{8}$ Lacy BE, Weiser KT, Kennedy AT, Crowell MD, Talley NJ. Functional dyspepsia: The economic impact to patients. Aliment Pharmacol Ther 2013; 38(2):170-177. PMID: 23725230.

9 Talley NJ, Vakil N, Practice Parameters Committee of the American College of Gastroenterology. Guidelines for the management of dyspepsia. Am J Gastroenterol 2005; 100(10):2324-2337. PMID: 16181387.

${ }^{10}$ Spiegel BMR, Farid M, van Oijen MGH, Laine L, Howden CW, Esrailian E. Adherence to best practice guidelines in dyspepsia: A survey comparing dyspepsia experts, community gastroenterologists and primary-care providers. Aliment Pharmacol Ther 2009; 29(8):871-881. PMID: 19183152.

${ }^{11}$ Cai JX, Campbell EJ, Richter JM. Concordance of outpatient esophagogastroduodenoscopy of the upper gastrointestinal tract with evidence-based guidelines. JAMA Intern Med 2015; 175(9):1563-1564. PMID: 26192987.

${ }_{12}$ Fiorenza JP, Tinianow AM, Chan WW. The initial management and endoscopic outcomes of dyspepsia in a low-risk patient population. Dig Dis Sci 2016; 6l(10):2942-2948. PMID: 26846116.

${ }^{13}$ Majumdar SR, Soumerai SB, Farraye FA, et al. Chronic acid-related disorders are common and underinvestigated. Am J Gastroenterol 2003; 98(11):2409-2414. PMID: 14638341.

14 US Centers for Disease Control and Prevention. Ambulatory Health Care Data. Statistics C-NCfH. 2019. https://www.cdc.gov/nchs/ahcd/index.htm. Accessed 2019/07/27,2019.

${ }^{15}$ Cerner. Lexicon Plus ${ }^{\circledast}$. Cerner Multum, Inc. https://www.cerner.com/ solutions/drug-database. Accessed 2019/09/13.

${ }^{16}$ Chey WD, Wong BC, Practice Parameters Committee of the American College of Gastroenterology. American College of Gastroenterology Guideline on the Management of Helicobacter pylori Infection. Am J Gastroenterol 2007; 102(8):1808-1825. PMID: 17608775.

${ }^{17}$ Levin TR, Schmittdiel JA, Kunz K, et al. Costs of acid-related disorders to a health maintenance organization. Am J Med 1997; 103(6):520-528. PMID: 9428836.

18 American Gastroenterological Association Medical Position Statement: Evaluation of Dyspepsia. Gastroenterology 1998; 114(3):579-581. PMID: 9496949.

${ }^{19}$ Axon AT. Chronic dyspepsia: Who needs endoscopy? Gastroenterology 1997; 112(4):1376-1380. PMID: 9098025.

${ }^{20}$ Ahlawat SK, Richard Locke G, Weaver AL, Farmer SA, Yawn BP, Talley NJ. Dyspepsia consulters and patterns of management: A population-based study. Aliment Pharmacol Ther 2005; 22(3):25l-259. PMID: 16091063.

${ }^{21}$ Sahai AV, Penman ID, Mishra G, et al. An assessment of the potential value of endoscopic ultrasound as a cost-minimizing tool in dyspeptic patients with persistent symptoms. Endoscopy 2001; 33(8):662-667. PMID: 11490381.

${ }^{22}$ Kurlander JE, Kolbe M, Rubenstein JH, et al. Internists' perceptions of proton pump inhibitor adverse effects and impact on prescribing practices: Results of a nationwide survey. Gastroenterology Res 2018; 11(1):11-17. PMID: 29511400.

${ }^{23}$ Cabana MD, Rand CS, Powe NR, et al. Why don't physicians follow clinical practice guidelines? A framework for improvement. JAMA 1999; 282(15):1458-1465. PMID: 10535437.

${ }^{24}$ Prior M, Guerin M, Grimmer-Somers K. The effectiveness of clinical guideline implementation strategies - A synthesis of systematic review findings. J Eval Clin Pract 2008; 14(5):888-897. PMID: 19018923.

${ }^{25}$ Valori RM, Brown CM, Strangeways P, Bradburn M. Reducing community dyspepsia drug costs: A controlled trial. Gut 2001; 49(4):495-501. PMID: 11559645 .

26 Elwyn G, Owen D, Roberts L, et al. Influencing referral practice using feedback of adherence to NICE guidelines: A quality improvement report for dyspepsia. Qual Saf Health Care 2007; 16(1):67-70. PMID: 17301208.

27 Menchik DA, Jin L. When do doctors follow patients' orders? Organizational mechanisms of physician influence. Soc Sci Res 2014; 48:171-184. PMID: 25l31283. 